\title{
Thermodynamic Properties of Liquids from Speed of Sound Measurements
}

\author{
M. Bijedić ${ }^{1 *}$, N. Neimarlija ${ }^{2}$ \\ ${ }^{1 *}$ Faculty of Technology, University of Tuzla, 8 Univerzitetska Str., 75000 Tuzla, Bosnia and Herzegovina \\ ${ }^{2}$ Faculty of Mechanical Engineering, University of Zenica, 1 Fakultetska Str., 72000 Zenica, Bosnia and Herzegovina \\ ${ }^{1}$ muhamed.bijedic@untz.ba
}

\begin{abstract}
A strategy for deriving thermodynamic properties of liquids $\left(\rho, c_{p}, c_{v}\right)$ from speed of sound is presented. It is based on numerical integration of differential equations connecting speed of sound with other thermodynamic properties. Two different procedures are recommended: One for liquids with lower and moderate critical pressures (LMCP), and another for liquids with higher critical pressures (HCP). The set of differential equations is solved as the initial value problem in both cases. However, for LMCP liquids initial conditions (several accurate values of $\rho$ and $c_{p}$ ) are specified along isobar near the critical pressure, and for HCP liquids along the liquid-vapor saturation curve. The procedures are tested on ethane, carbon dioxide, and water. The average absolute deviation of derived thermodynamic properties of ethane $\left(\rho, c_{p}, c_{v}\right)$, with respect to corresponding reference values, is $0.0014 \%$, $0.0741 \%$, and $0.1124 \%$, respectively. Corresponding deviations for carbon dioxide are $0.0008 \%, 0.0363 \%$, $0.0601 \%$, and for water $0.0001 \%, 0.0206 \%, 0.0289 \%$.
\end{abstract}

Keywords: Density; heat capacity; liquids; speed of sound.

\section{Introduction}

Several articles dealing with derivation of thermodynamic properties of liquids from experimental speeds of sound have been published in the last 40 years. An exact method of computing volume changes under high pressure from acoustic-wave-velocity was developed and its application illustrated with liquid mercury at pressures up to $1300 \mathrm{MPa}$ at temperatures $295.05 \mathrm{~K}, 313.65 \mathrm{~K}$, and 316.05 K (Davis and Gordon, 1967). An iterative method of calculation was used to determine thermodynamic properties of water from speed of sound in pressure range 0.1 to $350 \mathrm{MPa}$ and temperature range 251.15 to $293.15 \mathrm{~K}$ (Petitet et al., 1983). The effect of pressure on sound velocity and density of liquid toluene and $n$-heptane was investigated at pressures up to $260 \mathrm{MPa}$, in temperature ranges 173 to $320 \mathrm{~K}$ and 185 to $310 \mathrm{~K}$ (Muringer et al., 1985). Density, isothermal compressibility, isobaric thermal expansivity, and isobaric heat capacity were derived from speed of sound in liquid benzene and cyclohexane in temperature range 283 to $323 \mathrm{~K}$ and at pressures up to 170 and $80 \mathrm{MPa}$ respectively (Sun et al., 1987). Thermodynamic properties of liquid methanol and ethanol were derived from speed of sound at pressures up to 280 MPa in temperature ranges 203 to $263 \mathrm{~K}$ (Sun et al., 1990), and 193 to $263 \mathrm{~K}$ (Sun et al., 1991) respectively. Isentropic and isothermal compressibility of liquid $n$-hexane were derived from speed of sound at pressures up to $150 \mathrm{MPa}$, in temperature range 293 to $373 \mathrm{~K}$ (Daridon et al., 1998). Volumetric properties of liquid n-octadecane and $n$ nonadecane were derived from speed of sound at pressures up to $150 \mathrm{MPa}$, in temperature range 313 to $383 \mathrm{~K}$ (Dotour et al., 2000). A grid algorithm based on sound speed data was used to calculate thermodynamic properties of liquid $n$ dodecane in pressure range 0.1 to $140 \mathrm{MPa}$ and temperature range 293 to $433 \mathrm{~K}$ (Khasanshin et al., 2003). The acoustic data were combined with available values of density and isobaric heat capacity of water along one isobar at atmospheric pressure to calculate the same quantities over pressure range 0.1 to $90 \mathrm{MPa}$ and temperature range 274 to $394 \mathrm{~K}$ (Benedetto et al., 2005). A heuristic approach for the reduction of speed of sound data in the liquid region of water into a fundamental equation of state was proposed (Scalabrin et al., 2007). This technique provides an analytical formulation rather than local values of thermodynamic properties in temperature range 274 to 400 $\mathrm{K}$ and pressure range 0.09 to $100 \mathrm{MPa}$.

\section{Theory}

The density and the specific heat capacity at constant pressure of a liquid may be derived from its speed of sound if the following set of equations is solved (Benedetto et al., 2005):

$\left(\frac{\partial \rho}{\partial p}\right)_{T}=\frac{T \alpha_{p}^{2}}{c_{p}}+\frac{1}{u^{2}}$,

$\left(\frac{\partial c_{p}}{\partial p}\right)_{T}=-\frac{T}{\rho}\left[\alpha_{p}^{2}+\left(\frac{\partial \alpha_{p}}{\partial T}\right)_{p}\right]$,

$\alpha_{p}=-\frac{1}{\rho}\left(\frac{\partial \rho}{\partial T}\right)_{p}$,

where $p$ is the pressure, $T$ is the temperature, $u$ is the speed of sound, $\rho$ is the density, $c_{p}$ is the specific heat capacity at constant pressure, and $\alpha_{p}$ is the thermal expansion coefficient. The set of partial differential Eqs. (1) to (3) may be solved numerically in the range of $p$ and $T$ in which accurate speed of sound values are available. This set of 
equations may be solved as the initial value problem for the set of ordinary differential equations if temperature derivatives $(\partial \rho / \partial T)_{p}$ and $\left(\partial \alpha_{p} / \partial T\right)_{p}$ are known. The procedure of integration is stable if the initial values are specified at the lowest pressure of the range (Goodwin et al., 2003).

If subcritical pressures are considered, however, it is not possible to completely impose the initial values along the isobar at the lowest pressure of the range. Namely, decreasing the lower limit of the pressure range the temperature range also decreases. Therefore, in order to cover the maximum temperature range at subcritical pressures the initial values must be imposed at the highest pressure of the range. In this case, however, the procedure of integration will be stable only if the path of integration has a smaller slope than an isentrope passing through the area of interest. This condition excludes an isotherm as a possible choice for the path of integration. In $p-T$ domain lines parallel to the saturation line fulfill the upper condition. However, these lines cross the isotherm at the triple point prior the lower limit of the pressure range is reached, especially those far away from the saturation line. In order to avoid this, lines far away from the saturation line must have progressively bigger slope, but never exceed the slope of an isentrope. This may be achieved if the path of integration, $\phi$, passes through the points with temperature:

$T_{i}=T_{0}+\frac{i}{n}\left(T_{s}-T_{0}\right)$,

where $T_{0}$ is the lowest temperature of the range, $T_{s}$ is the saturation temperature, $i$ and $n$ are natural numbers. Now, recalling that (Sychev, 1983):

$\left(\frac{\partial z}{\partial x}\right)_{m}=\left(\frac{\partial z}{\partial x}\right)_{y}+\left(\frac{\partial z}{\partial y}\right)_{x}\left(\frac{\partial y}{\partial x}\right)_{m}$

Eqs. (1) and (2) become:

$$
\begin{aligned}
& \left(\frac{\partial \rho}{\partial p}\right)_{\phi}=\left(\frac{\partial \rho}{\partial T}\right)_{p}\left(\frac{\partial T}{\partial p}\right)_{\phi}+\frac{T \alpha_{p}^{2}}{c_{p}}+\frac{1}{u^{2}} \\
& \left(\frac{\partial c_{p}}{\partial p}\right)_{\phi}=\left(\frac{\partial c_{p}}{\partial T}\right)_{p}\left(\frac{\partial T}{\partial p}\right)_{\phi}-\frac{T}{\rho}\left[\alpha_{p}^{2}+\left(\frac{\partial \alpha_{p}}{\partial T}\right)_{p}\right] .
\end{aligned}
$$

The procedure of solving the set of Eqs. (3), (6), and (7) is virtually the same as the one for the set of Eqs. (1) to (3), with the only difference that the negative step of integration is used, and additional derivatives $(\partial T / \partial p)_{\phi}$ and $\left(\partial c_{p} / \partial T\right)_{p}$ have to be estimated. Therefore, the same set of initial values may be used for supercritical and subcritical pressure ranges.

Having calculated $\rho$ and $c_{p}$ in the pressure and temperature range of interest, the specific heat capacity at constant volume may be obtained from the equation:

$c_{v}=c_{p} u^{-2}\left(\frac{\partial \rho}{\partial p}\right)_{T}^{-1}$

The approach just described enables a wider temperature range, but at the cost of the initial values which are generally available with lower accuracy at higher pressures (especially $c_{p}$ ).

If the initial values are specified along the saturation line it would enable a wider temperature range than in the first approach. In the same time, almost all of the initial values would be specified at much lower pressures than in the second approach. This would also exclude all the temperature derivatives at constant pressure, and $c_{p}$ as well. The integration would take place along the isotherms like in the first approach, but the front of the integration would have shape of the saturation line rather than an isobar. For that reason it would be useful to introduce index $\sigma$ for all derivatives taken along the saturation line as well as $\alpha_{\sigma}$ and $c_{\sigma}$ instead of $\alpha_{p}$ and $c_{p}$, respectively. The useful relations are (Neindre and Vodar, 1975):

$\beta_{T}=\frac{\beta_{S} c_{\sigma}+(T / \rho) \alpha_{\sigma}\left(\alpha_{\sigma}+\beta_{S} \gamma_{\sigma}\right)}{c_{\sigma}-(T / \rho) \gamma_{\sigma}\left(\alpha_{\sigma}+\beta_{S} \gamma_{\sigma}\right)}$

$\beta_{S}=\left(u^{2} \rho\right)^{-1}$,

$\alpha_{\sigma}=-\frac{1}{\rho}\left(\frac{\partial \rho}{\partial T}\right)_{\sigma}$

$\gamma_{\sigma}=\left(\frac{\partial p}{\partial T}\right)_{\sigma}$

$c_{p}=c_{\sigma}+\frac{T}{\rho} \alpha_{p} \gamma_{\sigma}$

$\alpha_{p}=\alpha_{\sigma}+\beta_{T} \gamma_{\sigma}$

$c_{v}=c_{\sigma}-\frac{T}{\rho} \frac{\alpha_{\sigma} \alpha_{p}}{\beta_{T}}$.

Combining Eqs. (1) to (3) and (9) to (15), with assistance of differential calculus, the following equations are obtained:

$\left(\frac{\partial \rho}{\partial p}\right)_{T}=\beta_{T} \rho$

$\left(\frac{\partial c_{\sigma}}{\partial p}\right)_{T}=-\frac{T}{\rho}\left[\alpha_{p}^{2}+\left(\frac{\partial \alpha_{p}}{\partial T}\right)_{\sigma}\right]+\frac{1}{\rho} \alpha_{p} \beta_{T} \gamma_{\sigma}$.

In order to solve Eqs. (16) and (17) for $\rho$ and $c_{\sigma}$ it is necessary to specify their initial values along the saturation line. However, $c_{\sigma}$ is not experimental quantity and it has to be calculated from $c_{p}$. Derivatives $(\partial p / \partial T)_{\sigma}$ and $(\partial \rho / \partial T)_{\sigma}$ have to be estimated in the first place, and then $\beta_{S}$ and $\alpha_{\sigma}$ are calculated from (10) and (11), respectively. Now, (13) and (14) are combined to eliminate $\alpha_{p}$. Then, Eq. (9) is reconfigured into the following form:

$\left(\frac{T}{\rho} \gamma_{\sigma}^{2}\right) \beta_{T}^{2}+\left(2 \frac{T}{\rho} \alpha_{\sigma} \gamma_{\sigma}-c_{p}\right) \beta_{T}+\frac{T}{\rho} \alpha_{\sigma}^{2}+c_{p} \beta_{S}=0$,

and solved for $\beta_{T}$. Finally, $\alpha_{p}$ is calculated from (14), and $c_{\sigma}$ from (13). Having estimated $\left(\partial \alpha_{p} / \partial T\right)_{\sigma}$, a numerical procedure may be applied and Eqs. (16) and (17) solved for $\rho$ and $c_{\sigma}$ in the range of $p$ and $T$ in which accurate values of 
$u$ are available. It should be noted that Eq. (18) is not used anymore, and from this point on $\beta_{T}$ is calculated from (9).

\section{Results}

\subsection{Ethane}

Domain of integration for ethane is shown at Figure 1.

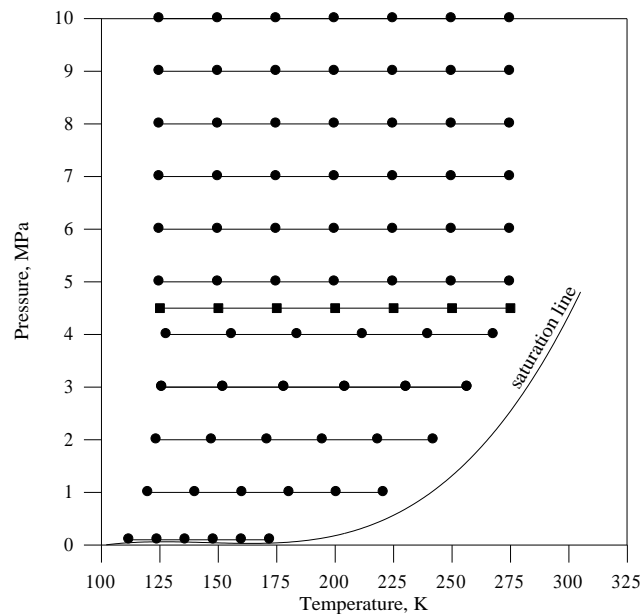

Figure 1. Domain of integration for ethane; $\mathbf{\square}$ speed of sound + initial values; speed of sound.

\subsubsection{Supercritical pressures}

The initial values of $\rho$ and $c_{p}$ were specified at the isobar of $4.5 \mathrm{MPa}$, in the temperature range 125 to $275 \mathrm{~K}$, and the integration was performed up to $10 \mathrm{MPa}$. The pressure range was divided into seven isobars $(4.5,5,6,7$, 8,9 , and $10 \mathrm{MPa}$ ), while the temperature range was divided into seven isotherms $(125,150,175,200,225,250$, and 275 $\mathrm{K})$. The initial values, the reference values, as well as the sound-speed values were obtained from the fundamental equation of state (EOS) for ethane (Friend et al., 1991). The sound-speed values between the isobars were estimated from a Lagrangian interpolation polynomial of the sixthdegree with respect to pressure. Temperature derivatives $(\partial \rho / \partial T)_{p}$ and $\left(\partial \alpha_{p} / \partial T\right)_{p}$ were estimated analytically from respective functions:

$\rho=\frac{a+b T+c T^{2}}{1+d T+e T^{2}+f T^{3}}, \alpha_{p}=\frac{a+b T+c T^{2}}{1+d T+e T^{2}+f T^{3}}$,

in the pressure range 4.5 to $5 \mathrm{MPa}$, and from a Lagrangian interpolation polynomial of the sixth-degree in the pressure range 5 to $10 \mathrm{MPa}$. Parameters $a, b, c, d$, e, and $f$ in Eqs. (19) were obtained by solving a linear least-squares problem with iterative refinement of Björck (Golub and Van Loan, 1983). The numerical integration of Eqs. (1) and (2), with respect to pressure, was performed by implicit multistep Adams-Moulton method with adaptive step-size (Chenney and Kincaid, 1985).

Relative deviations of $\rho, c_{p}$, and $c_{v}$, with respect to corresponding reference values, are given at Figures 2, 3, and 4 , respectively. The average absolute deviation of density is $0.0007 \%$, and the maximum relative deviation is $+0.0132 \% /-0.0015 \%$. The average absolute deviation of $c_{p}$ is $0.0915 \%$, and the maximum relative deviation is $+0.0152 \% /-1.0657 \%$. The average absolute deviation of $c_{v}$ is $0.1155 \%$, and the maximum relative deviation is $+0.4293 \% /-0.5302 \%$.

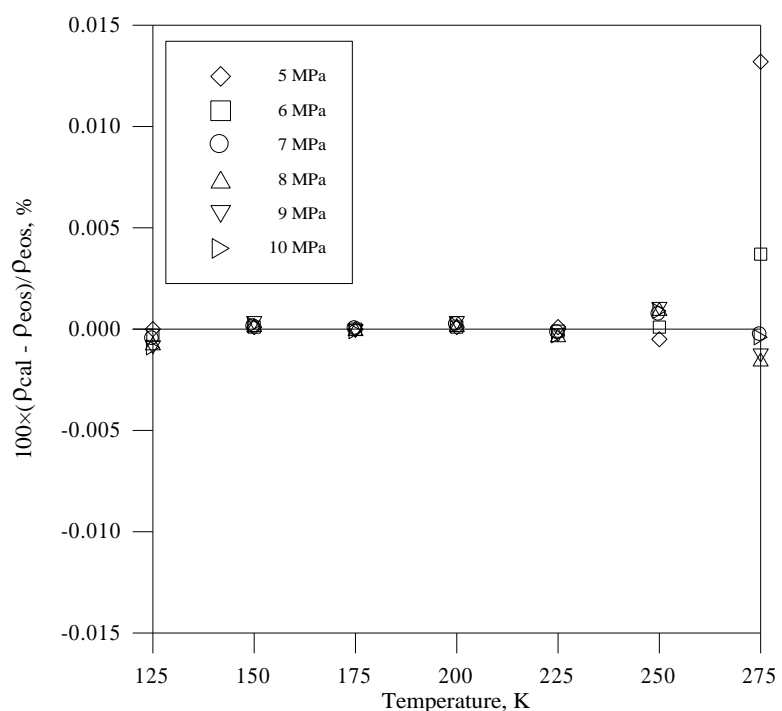

Figure 2. Relative deviation of ethane density with respect to reference values (Friend et al., 1991).

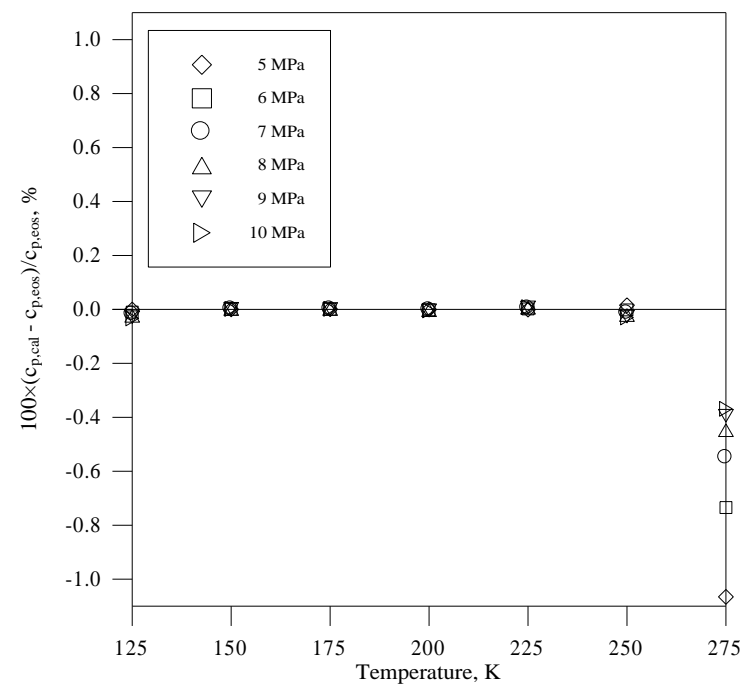

Figure 3. Relative deviation of ethane $c_{p}$ with respect to reference values (Friend et al., 1991).

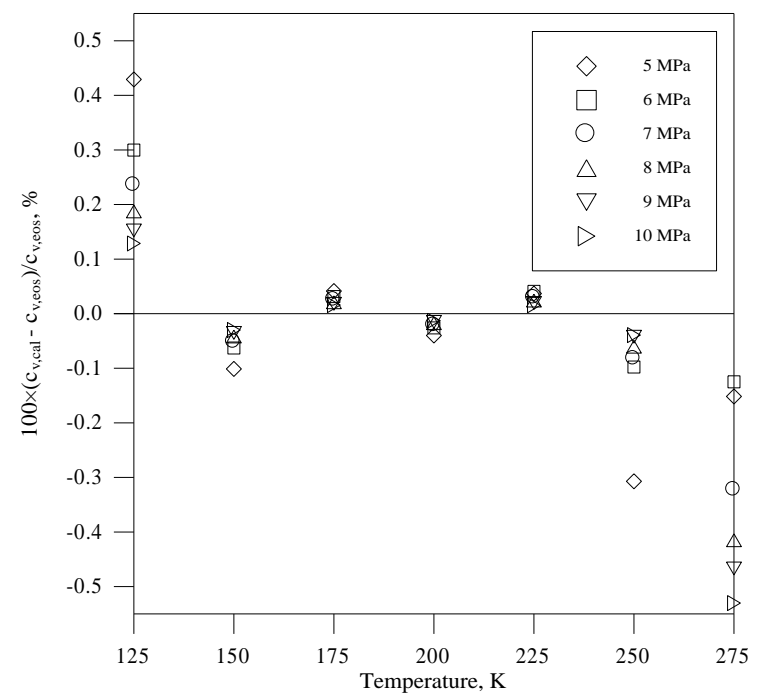

Figure 4. Relative deviation of ethane $c_{v}$ with respect to reference values (Friend et al., 1991).

\subsubsection{Subcritical pressures}

The numerical integration of Eqs. (6) and (7) was performed in the pressure range 4.5 to $0.1 \mathrm{MPa}$, along the 
paths connecting the points with temperature $T_{i}=100+\frac{i}{7}\left(T_{s}-100\right)$, where $i=1,2,3,4,5$, and 6 . The initial values of $\rho$ and $c_{p}$ were specified at the isobar of 4.5 $\mathrm{MPa}$. The pressure range was divided into six isobars (4.5, 4, 3, 2, 1, and 0.1 MPa). The initial values, the reference values, as well as the sound-speed values were obtained from the fundamental EOS for ethane (Friend et al., 1991). The sound-speed values between the isobars were obtained from a Lagrangian interpolation polynomial of the fifthdegree with respect to pressure. Temperature derivatives $(\partial \rho / \partial T)_{p},\left(\partial c_{p} / \partial T\right)_{p}$, and $\left(\partial \alpha_{p} / \partial T\right)_{p}$ were estimated analytically from respective functions:

$$
\begin{aligned}
& \rho=\frac{a+b T+c T^{2}}{1+d T+e T^{2}+f T^{3}}, \\
& c_{p}=\frac{a+b T+c T^{2}}{1+d T+e T^{2}+f T^{3}}, \\
& \alpha_{p}=\frac{a+b T+c T^{2}}{1+d T+e T^{2}+f T^{3}},
\end{aligned}
$$

in the pressure range 4.5 to $3 \mathrm{MPa}$, and from a Lagrangian interpolation polynomial of the fifth-degree in the pressure range 3 to $0.1 \mathrm{MPa}$. Parameters $a, b, c, d, e$, and $f$ in Eqs. (20) were obtained by solving a linear least-squares problem with iterative refinement of Björck (Golub and Van Loan, 1983). Pressure derivatives $(\partial T / \partial p)_{\phi}$ were estimated from a Lagrangian interpolation polynomial of the fifth-degree. The numerical integration of Eqs. (6) and (7), with respect to pressure, was performed by implicit multistep Adams-Moulton method with adaptive step-size (Chenney and Kincaid, 1985).

Relative deviations of $\rho, c_{p}$, and $c_{v}$, with respect to corresponding reference values are given at Figures 5, 6, and 7 , respectively. The average absolute deviation of density is $0.0021 \%$, and the maximum relative deviation is $+0.0243 \% /-0.0029 \%$. The average absolute deviation of $c_{p}$ is $0.0567 \%$, and the maximum relative deviation is $+0.4893 \% /-0.0465 \%$. The average absolute deviation of $c_{v}$ is $0.1093 \%$, and the maximum relative deviation is $+0.5113 \% /-0.1046 \%$. See Appendix for the uncertainties originating from EOSs used.

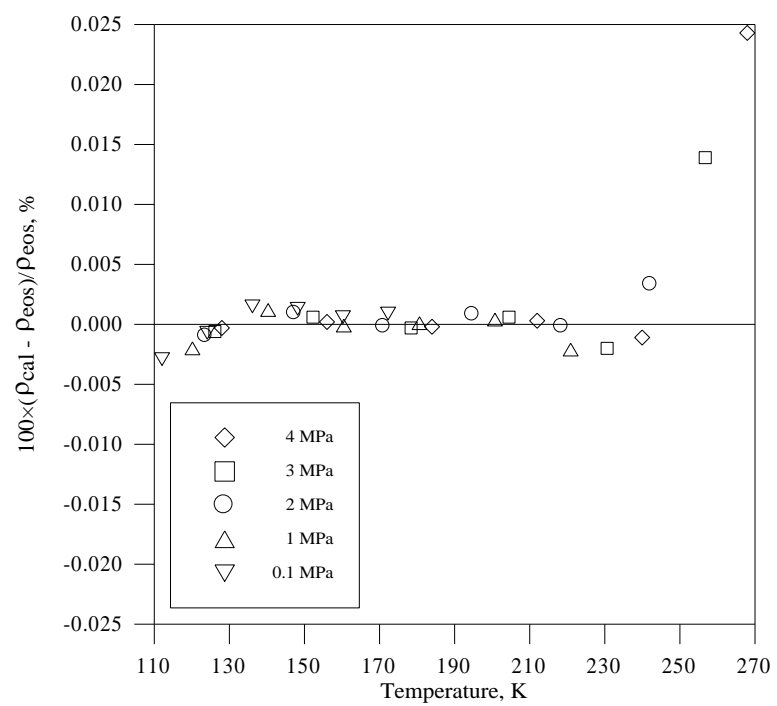

Figure 5. Relative deviation of ethane density with respect to reference values (Friend et al., 1991).

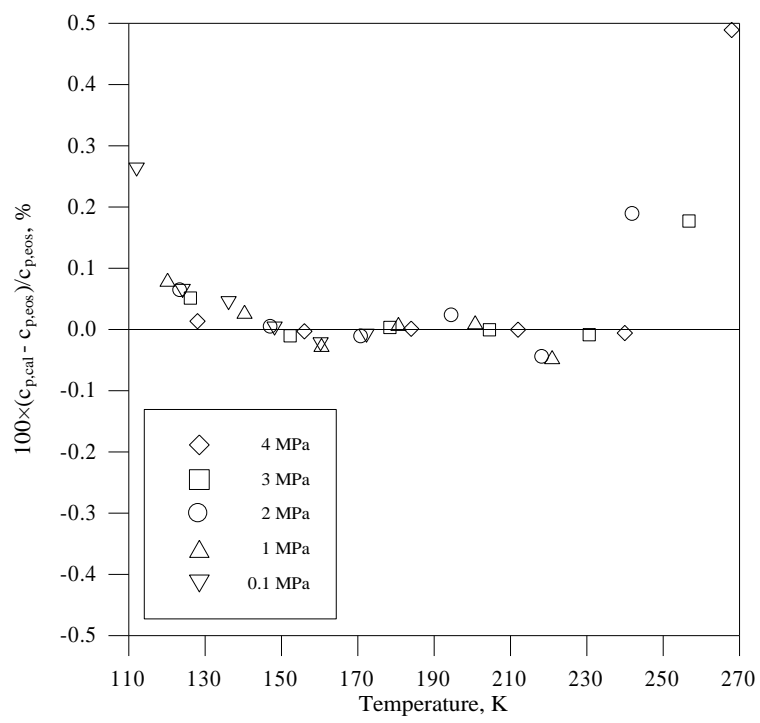

Figure 6. Relative deviation of ethane $c_{p}$ with respect to reference values (Friend et al., 1991).

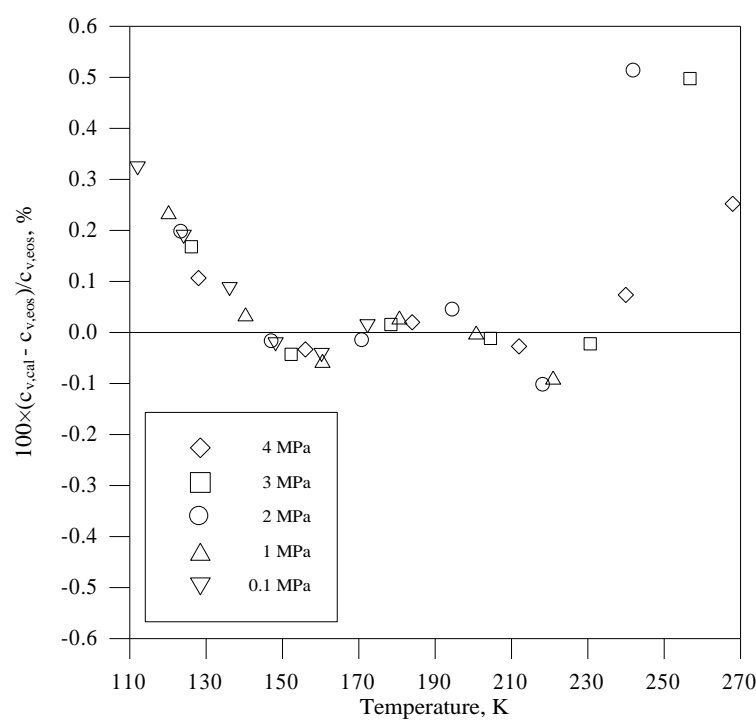

Figure 7. Relative deviation of ethane $c_{v}$ with respect to reference values (Friend et al., 1991).

\subsection{Carbon dioxide}

Domain of integration for $\mathrm{CO}_{2}$ is shown at Figure 8 .

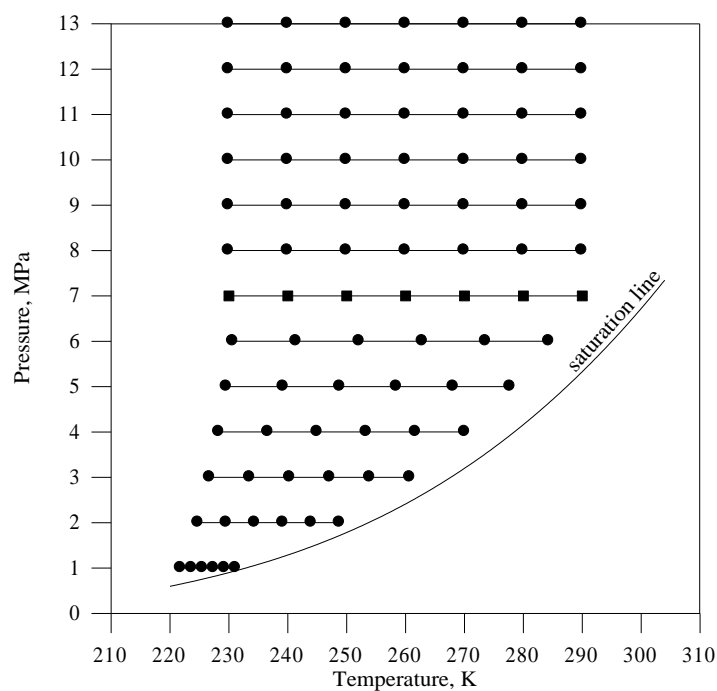

Figure 8. Domain of integration for $\mathrm{CO}_{2} ; \mathbf{\square}$ speed of sound + initial values; - speed of sound. 


\subsubsection{Supercritical pressures}

The initial values of $\rho$ and $c_{p}$ were specified at the isobar of $7 \mathrm{MPa}$, in the temperature range 230 to $290 \mathrm{~K}$, and the integration was performed up to $13 \mathrm{MPa}$. The pressure range was divided into seven isobars $(7,8,9,10$, 11,12 , and $13 \mathrm{MPa}$ ), while the temperature range was divided into seven isotherms $(230,240,250,260,270,280$, and $290 \mathrm{~K}$ ). The initial values, the reference values, as well as the sound-speed values were obtained from the fundamental EOS for $\mathrm{CO}_{2}$ (Span and Wagner, 1996). The sound-speed values between the isobars were estimated from a Lagrangian interpolation polynomial of the sixthdegree with respect to pressure. Temperature derivatives $(\partial \rho / \partial T)_{p}$ and $\left(\partial \alpha_{p} / \partial T\right)_{p}$ were estimated analytically from

Eqs. (19) in the pressure range 7 to $12 \mathrm{MPa}$, and from a Lagrangian interpolation polynomial of the sixth-degree in the pressure range 12 to $13 \mathrm{MPa}$. Parameters $a, b, c, d$, e, and $f$ in Eqs. (19) were obtained by solving a linear leastsquares problem with iterative refinement of Björck (Golub and Van Loan, 1983). The numerical integration of Eqs. (1) and (2), with respect to pressure, was performed by implicit multistep Adams-Moulton method with adaptive step-size (Chenney and Kincaid, 1985).

Relative deviations of $\rho, c_{p}$, and $c_{v}$, with respect to corresponding reference values, are given at Figures 9, 10, and 11 , respectively. The average absolute deviation of density is $0.0003 \%$, and the maximum relative deviation is $+0.0010 \% /-0.0007 \%$. The average absolute deviation of $c_{p}$ is $0.0040 \%$, and the maximum relative deviation is $+0.0199 \% /-0.0252 \%$. The average absolute deviation of $c_{v}$ is $0.0186 \%$, and the maximum relative deviation is $+0.2258 \% /-0.0512 \%$.

\subsubsection{Subcritical pressures}

The numerical integration of Eqs. (6) and (7) was performed in the pressure range 7 to $1 \mathrm{MPa}$, along the paths connecting the points with temperature

$T_{i}=220+\frac{i}{7}\left(T_{s}-220\right)$,

where $i=1,2,3,4,5$, and 6 . The initial values of $\rho$ and $c_{p}$ were specified at the isobar of $7 \mathrm{MPa}$. The pressure range

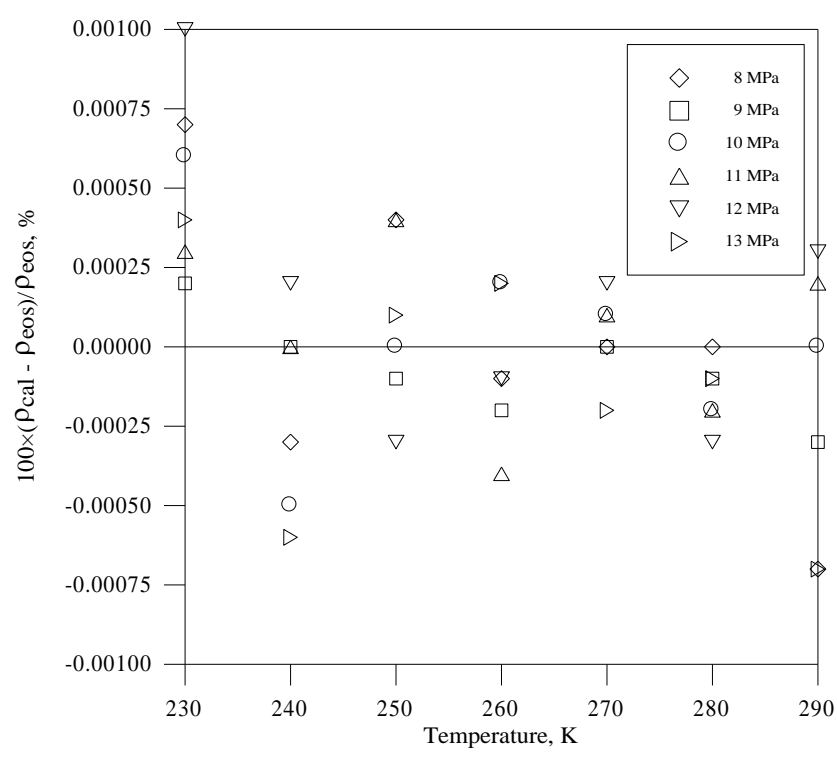

Figure 9. Relative deviation of $\mathrm{CO}_{2}$ density with respect to reference values (Span and Wagner, 1996).

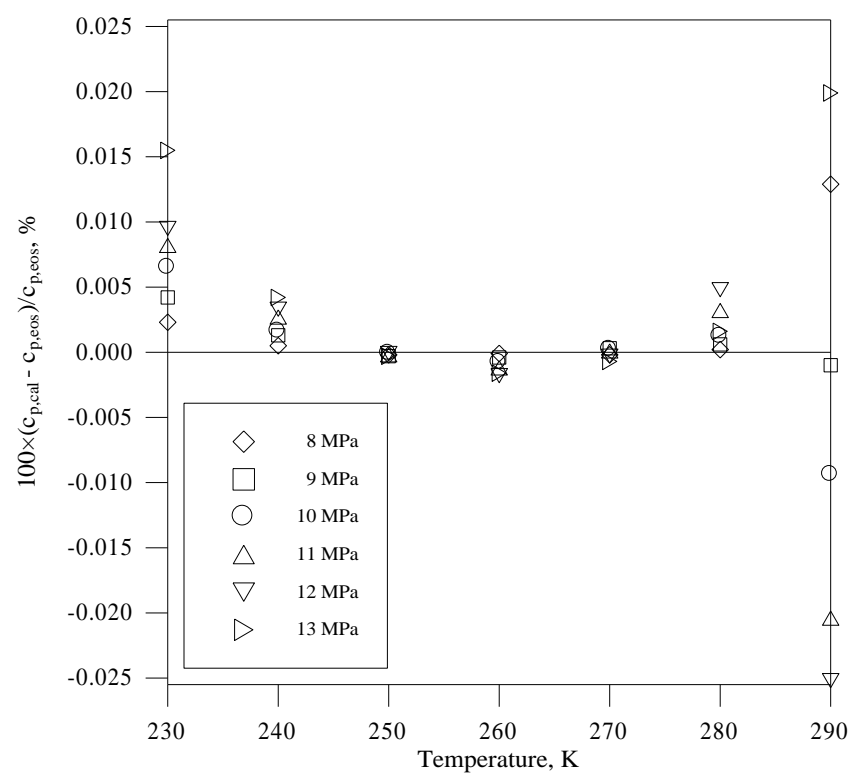

Figure 10. Relative deviation of $\mathrm{CO}_{2} c_{p}$ with respect to reference values (Span and Wagner, 1996).

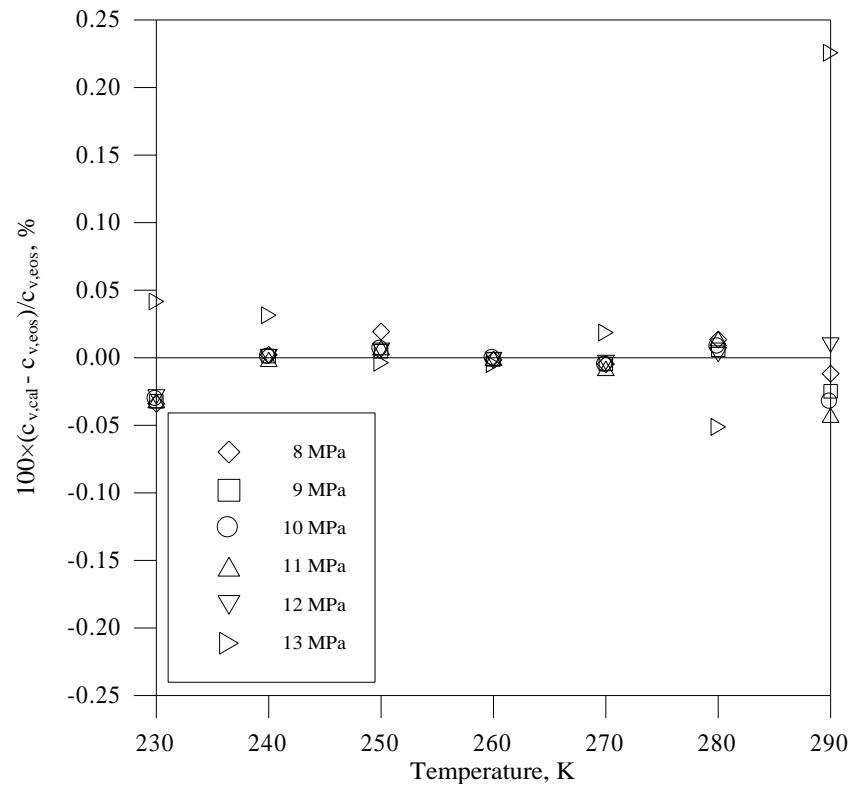

Figure 11. Relative deviation of $\mathrm{CO}_{2} c_{v}$ with respect to reference values (Span and Wagner, 1996).

was divided into seven isobars $(7,6,5,4,3,2$, and $1 \mathrm{MPa})$. The initial values, the reference values, as well as the sound-speed values were obtained from the fundamental EOS for $\mathrm{CO}_{2}$ (Span and Wagner, 1996). The sound-speed values between the isobars were obtained from a Lagrangian interpolation polynomial of the sixth-degree with respect to pressure. Temperature derivatives $(\partial \rho / \partial T)_{p}$ , $\left(\partial c_{p} / \partial T\right)_{p}$, and $\left(\partial \alpha_{p} / \partial T\right)_{p}$ were estimated analytically from Eqs. (20) in the pressure range 7 to $4 \mathrm{MPa}$, and from a Lagrangian interpolation polynomial of the fifth-degree in the pressure range 4 to $1 \mathrm{MPa}$. Parameters $a, b, c, d, e$, and $f$ in Eqs. (20) were obtained by solving a linear least-squares problem with iterative refinement of Björck (Golub and Van Loan, 1983). Pressure derivatives $(\partial T / \partial p)_{\phi}$ were estimated from a Lagrangian interpolation polynomial of the sixth-degree. The numerical integration of Eqs. (6) and (7), with respect to pressure, was performed by implicit 
multistep Adams-Moulton method with adaptive step-size (Chenney and Kincaid, 1985).

Relative deviations of $\rho, c_{p}$, and $c_{v}$, with respect to corresponding reference values are given at Figures 12, 13, and 14, respectively. The average absolute deviation of density is $0.0013 \%$, and the maximum relative deviation is $+0.0042 \% /-0.0086 \%$. The average absolute deviation of $c_{p}$ is $0.0686 \%$, and the maximum relative deviation is $+0.2667 \% /-0.5460 \%$. The average absolute deviation of $c_{v}$ is $0.1016 \%$, and the maximum relative deviation is $+0.8542 \% /-0.5692 \%$. See Appendix for the uncertainties originating from EOSs used.

\subsection{Water}

Initial values of $\rho$ and $c_{p}$ were specified along the saturation line, in the temperature range 372.756 to 537.091 $\mathrm{K}$ (which corresponds to the pressure range 0.1 to $5 \mathrm{MPa}$ ). The temperature range was divided into seven isotherms (372.756, 424.981, 453.028, 485.527, 507.003, 523.504, and $537.091 \mathrm{~K}$ ), along which the integration was performed. The initial values, the reference values, as well

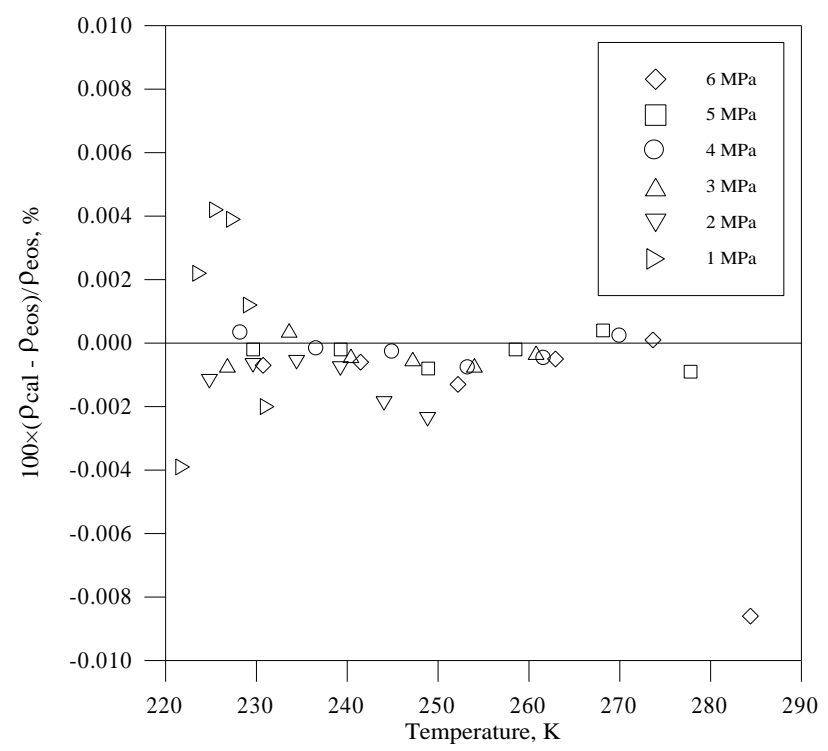

Figure 12. Relative deviation of $\mathrm{CO}_{2}$ density with respect to reference values (Span and Wagner, 1996).

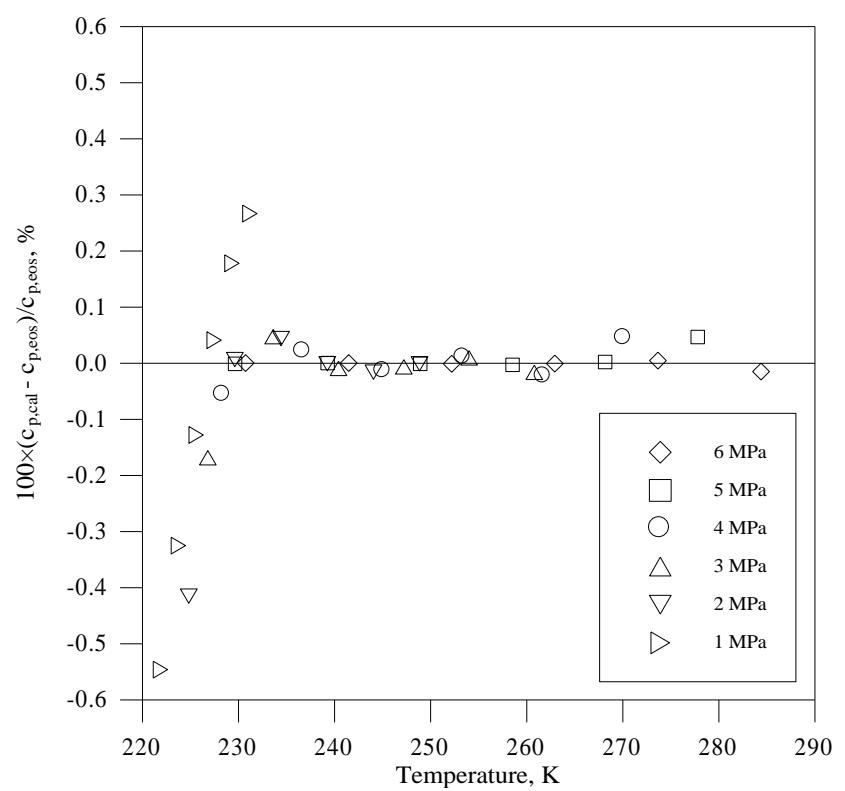

Figure 13. Relative deviation of $\mathrm{CO}_{2} c_{p}$ with respect to reference values (Span and Wagner, 1996).

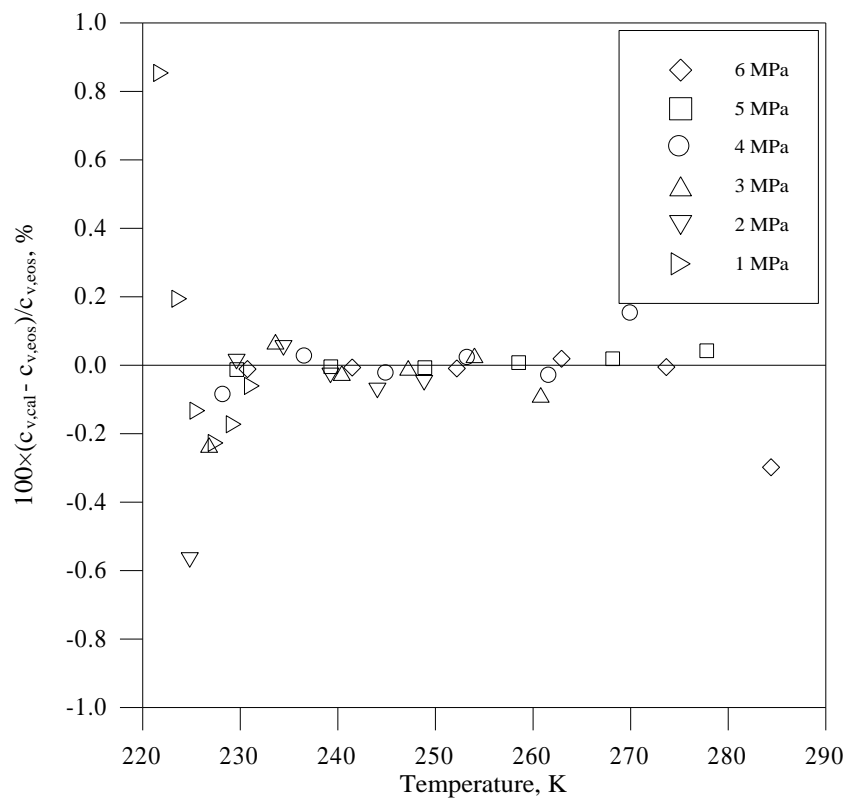

Figure 14. Relative deviation of $\mathrm{CO}_{2} c_{v}$ with respect to reference values (Span and Wagner, 1996).

as the sound-speed values were obtained from the fundamental EOS for water (Wagner and Pruss, 2002). The sound-speed values were specified along the isotherms at pressure increments of $5 \mathrm{MPa}$, ending at $30.1 \mathrm{MPa}$ at the lowest temperature and $35 \mathrm{MPa}$ at the highest temperature. Between the isobars sound-speed values were estimated from a Lagrange interpolation polynomial of the sixthdegree with respect to pressure. Derivatives $(\partial p / \partial T)_{\sigma}$, $(\partial \rho / \partial T)_{\sigma}$, and $\left(\partial \alpha_{p} / \partial T\right)_{\sigma}$ were estimated analytically from respective functions:

$\ln p=\frac{a+b T+c T^{2}}{1+d T+e T^{2}+f T^{3}}$,

$\ln \rho=\frac{a+b T+c T^{2}}{1+d T+e T^{2}+f T^{3}}$,

$\ln \alpha_{p}=\frac{a+b T+c T^{2}}{1+d T+e T^{2}+f T^{3}}$.

Parameters $a, b, c, d, e$, and $f$ in Eqs. (21) were obtained by solving a linear least-squares problem with iterative refinement of Björck (Golub and Van Loan, 1983). Numerical integration of Eqs. (16) and (17) was performed by Verner's, adaptive step-size Runge-Kutta method of the sixth-order approximation (Ralston and Wilf, 1960). Domain of integration for water is shown at Figure 15.

Relative deviations of $\rho, c_{p}$, and $c_{v}$, with respect to corresponding reference values, are given at Figures 16, 17 , and 18 , respectively. The average absolute deviation of density is $0.0001 \%$, and the maximum relative deviation is $+0.0007 \% /-0.0001 \%$. The average absolute deviation of $c_{p}$ is $0.0206 \%$, and the maximum relative deviation is $+0.0098 \% /-0.1003 \%$. The average absolute deviation of $c_{v}$ is $0.0289 \%$, and the maximum relative deviation is $+0.0639 \% /-0.1260 \%$. See Appendix for the uncertainties originating from EOSs used. 


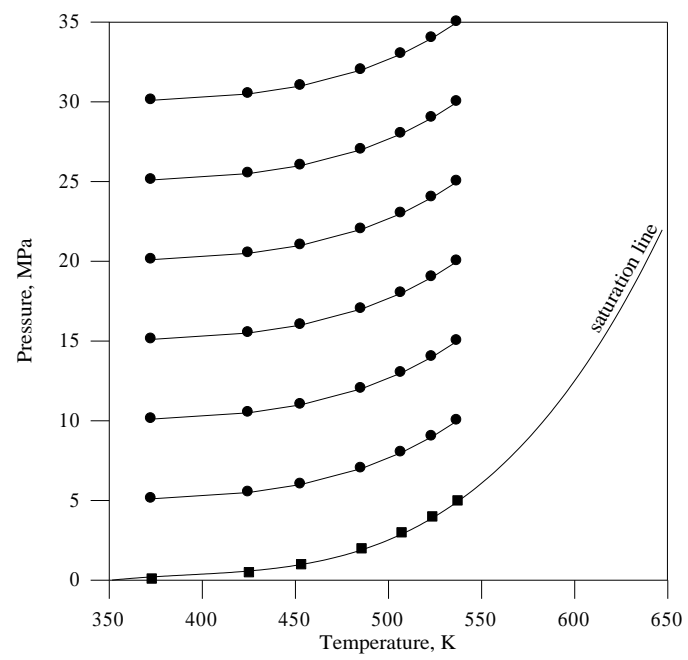

Figure 15. Domain of integration for water; $\mathbf{\square}$ speed of sound + initial values; speed of sound.

\section{Conclusions}

Presented strategy for deriving thermodynamic properties from speed of sound is consisted of two entirely new procedures that have never been investigated before. It may give satisfactory results for majority of technically interesting liquids in the pressure range from the triple point to far above the critical point, and the temperature range from the triple point to near the critical point. For substances with lower and moderate critical pressures initial values are specified along isobare near the critical pressure, and for those with higher critical pressures along the liquidvapor saturation curve. In both cases just a few accurate values of $\rho$ and $c_{p}$ will be sufficient.

\section{Appendix}

The uncertainties originating from EOSs used are as follows:

Ethane: The uncertainties of the equation of state are $0.2 \%$ in density, $2.5 \%$ in isobaric heat capacity above $150 \mathrm{~K}$, and $1.5 \%$ in isochoric heat capacity above $150 \mathrm{~K}$. The uncertainty in speed of sound ranges from $0.6 \%$ in the liquid and vapor below the critical temperature to less than $2 \%$ elsewhere, except in the critical region.

Carbon dioxide: At pressures up to $30 \mathrm{MPa}$ and temperatures up to $523 \mathrm{~K}$, the estimated uncertainty ranges from $0.03 \%$ to $0.05 \%$ in density, $0.03 \%$ (in the vapor) to $1 \%$ in the speed of sound ( $0.5 \%$ in the liquid) and $0.15 \%$ (in the vapor) to $1.5 \%$ (in the liquid) in heat capacity. Special interest has been focused on the description of the critical region and the extrapolation behavior of the formulation (to the limits of chemical stability).

Water: The uncertainty in density of the equation of state is $0.0001 \%$ at $0.1 \mathrm{MPa}$ in the liquid phase, and $0.001 \%$ at other liquid states at pressures up to $10 \mathrm{MPa}$ and temperatures to $423 \mathrm{~K}$. In the vapor phase, the uncertainty is $0.05 \%$ or less. The uncertainties rise at higher temperatures and/or pressures, but are generally less than $0.1 \%$ in density except at extreme conditions. The uncertainty in pressure in the critical region is $0.1 \%$. The uncertainty of the speed of sound is $0.15 \%$ in the vapor and $0.1 \%$ or less in the liquid, and increases near the critical region and at high temperatures and pressures. The

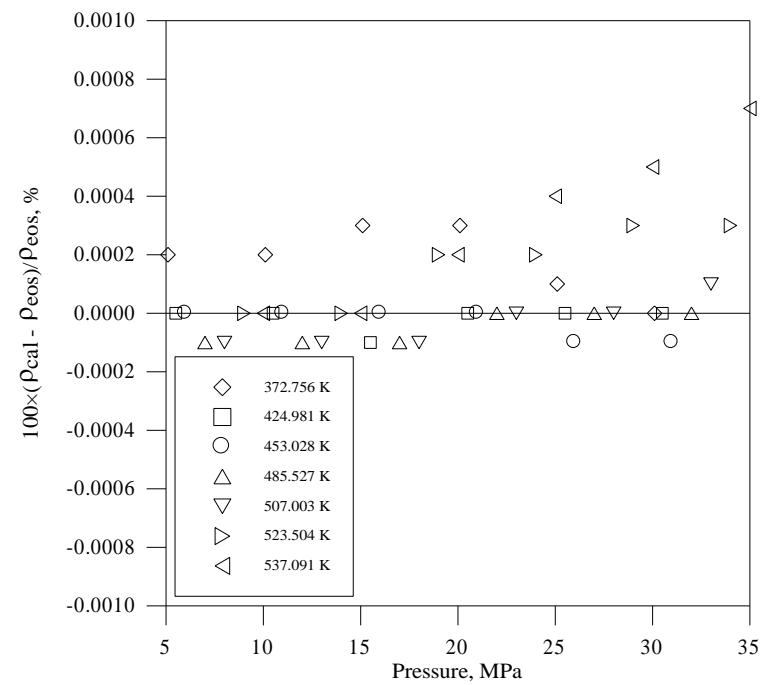

Figure 16. Relative deviation of water density with respect to reference values (Wagner and Pruss, 2002).

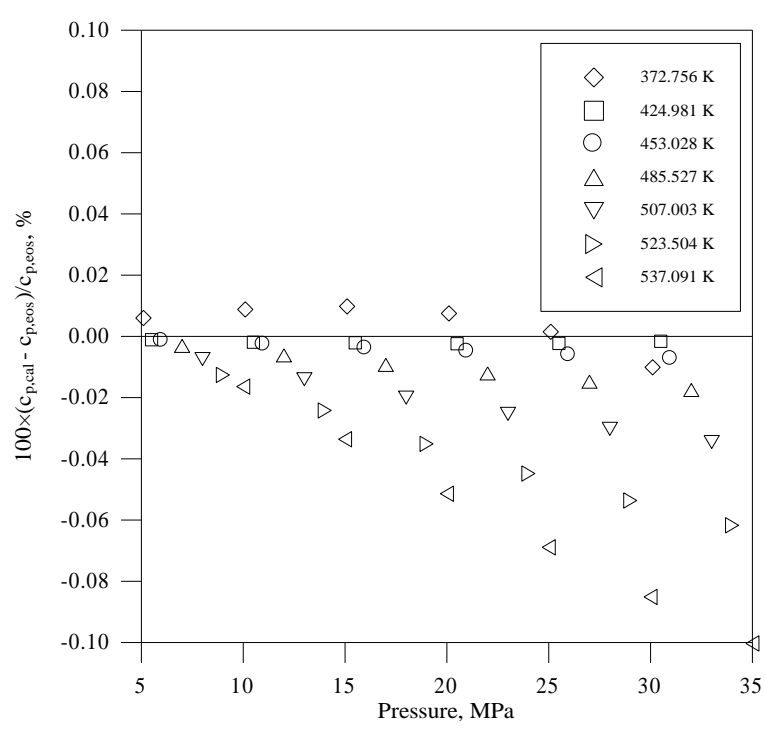

Figure 17. Relative deviation of water $c_{p}$ with respect to reference values (Wagner and Pruss, 2002).

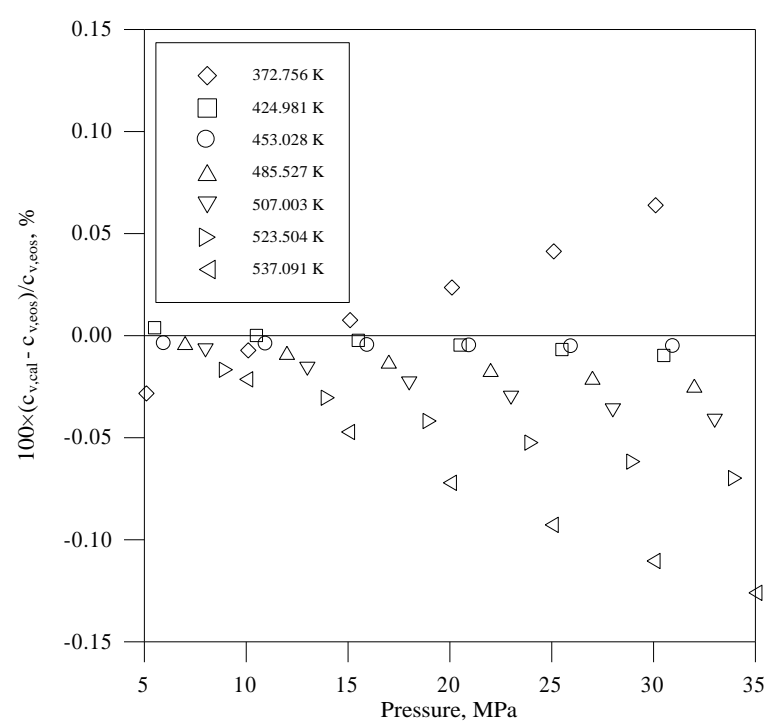

Figure 18. Relative deviation of water $c_{v}$ with respect to reference values (Wagner and Pruss, 2002).

uncertainty in isobaric heat capacity is $0.2 \%$ in the vapor and $0.1 \%$ in the liquid, with increasing values in the critical region and at high pressures. The uncertainties of saturation 
conditions are $0.025 \%$ in vapor pressure, $0.0025 \%$ in saturated liquid density, and $0.1 \%$ in saturated vapor density. The uncertainties in the saturated densities increase substantially as the critical region is approached.

\section{References:}

Benedetto, G., Gavioso, R. M., Giuliano Albo, P. A., Lago, S., Madonna Ripa, D., Spagnolo, R., 2005, "Speed of Sound in Pure Water at Temperatures between 274 and 394 $\mathrm{K}$ and at Pressures up to $90 \mathrm{MPa}$ ", Int. J. Thermophys., Vol. 26, No. 6, pp. 1667-1680.

Chenney, W., Kincaid, D., 1985, Numerical Mathematics and Computing, Brooks/Cole Publishing Company, Pacific Grove, California.

Daridon, J. L., Lagourette, B., Grolier, J. -P. E., 1998, "Experimental Measurements of the Speed of Sound in $n$ Hexane from 293 to $373 \mathrm{~K}$ and up to $150 \mathrm{MPa}$, Int. J. Thermophys., Vol. 19, No. 1, pp. 145-160.

Davis, L. A., Gordon, R. B., 1967, "Compression of Mercury at High Pressure", J. Chem. Physics, Vol. 46, pp. 2650-2660.

Dotour, S., Daridon, J. L., Lagourette, B., 2000, "Pressure and Temperature Dependence of the Speed of Sound and Related Properties in Normal Octadecane and Nonadecane", Int. J. Thermophys., Vol. 21, No. 1, pp. $173-$ 184.

Friend, D. G., Ingham, H., Ely, J. F., 1991, "Thermophysical Properties of Ethane", J. Phys. Chem. Ref. Data, Vol. 20, No. 2, pp. 275-347.

Golub, G. H., Van Loan, C. F., 1983, Matrix Computations, Johns Hopkins University Press, Baltimore, Maryland.

Goodwin, A. R. H., Marsh, K. N., Wakeham, W. A., (Ed.), 2003, Measurement of the Thermodynamic Properties of Single Phases, Elsevier, Amsterdam.

Khasanshin, T. S., Shchamialiou, A. P., Poddubskij, O. G., 2003, "Thermodynamic Properties of Heavy $n$-Alkanes in the Liquid State: $n$-Dodecane", Int. J. Thermophys., Vol 24, No. 5, pp. 1277-1289.

Le Neindre, B., Vodar, B., (Ed.), 1975, Experimental Thermodynamics Volume II: Experimental Thermodynamics of Non-reacting Fluids, Butterworths, London.
Muringer, M. J. P., Trappeniers, N. J., Biswas, S. N., 1985, "The Effect of Pressure on the Sound Velocity and Density of Toluene and $n$-Heptane up to 2600 bar", Phys. Chem. Liq., Vol 14, No. 4, pp.273-296.

Petitet, J. P., Tufeu, R., Le Neindre, B., 1983, "Determination of the Thermodynamic Properties of Water from Measurements of the Speed of Sound in the Temperature Range 251.15-293.15 K and the Pressure Range 0.1-350 MPa”, Int. J. Thermophys., Vol. 4, No. 1, pp. 35-50.

Ralston, A., Wilf, H. S., 1960, Mathematical Methods for Digital Computers, John Wiley and Sons, New York.

Scalabrin, G., Concion, M. G., Marchi, P., Lago, S., 2007, "Development of a Fundamental Equation of State for the Liquid Region of a Pure Fluid from Speed of Sound Measurements", Experimental Thermal and Fluid Science, Vol. 31, No. 6, pp. 539-549.

Span, R., Wagner, W., 1996, “A New Equation of State for Carbon Dioxide Covering the Fluid Region from the TriplePoint Temperature to $1100 \mathrm{~K}$ at Pressures up to $800 \mathrm{MPa}$ ", J. Phys. Chem. Ref. Data, Vol. 25, No. 6, pp. 1509-1596.

Sun, T. F., Kortbeek, P. J., Trappeniers, N. J., Biswas, S. N., 1987, "Acoustic and Thermodynamic Properties of Benzene and Cyclohexane as a Function of Pressure and Temperature", Phys. Chem. Liq., Vol. 16, No. 3, pp. 163178.

Sun, T. F., Schouten, J. A., Biswas, S. N., 1990, "Determination of the Thermodynamic Properties of Liquid Methanol from 203 to $263 \mathrm{~K}$ and up to $280 \mathrm{MPa}$ from Speed of Sound Measurements", Ber. Bunsen. Phys. Chem., Vol. 94, No. 4, pp. 528-534.

Sun, T. F., Schouten, J. A., Biswas, S. N., 1991, "Determination of the Thermodynamic Properties of Liquid Ethanol from 193 to $263 \mathrm{~K}$ and up to $280 \mathrm{MPa}$ from Speed of Sound Measurements", Int. J. Thermophys., Vol 12, No. 2, pp. 381-395.

Sychev, V. V., 1983, The Differential Equations of Thermodynamics, Mir Publishers, Moscow.

Wagner. W., Pruss, A., 2002, "The IAPWS Formulation 1995 for the Thermodynamic Properties of Ordinary Water Substance for General and Scientific Use", J. Phys. Chem. Ref. Data, Vol. 31, No. 2, pp. 387-535. 